\title{
Why do pitched horizontal lines have such a small effect on visually perceived eye level?
}

\author{
ROBERT B. POST \\ University of California, Davis, California \\ ROBERT B. WELCH \\ NASA-Ames Research Center, Mountain View, California \\ and \\ VALERIE D. CLARK \\ University of Califormia, Davis, Califormia
}

\begin{abstract}
In two experiments, visually perceived eye level (VPEL) was measured while subjects viewed twodimensional displays that were either upright or pitched $20^{\circ}$ top-toward or $20^{\circ}$ top-away from them. In Experiment 1, it was demonstrated that binocular exposure to a pair of pitched vertical lines or to a pitched random dot pattern caused a substantial upward VPEL shift for the top-toward pitched array and a similarly large downward shift for the top-away array. On the other hand, the same pitches of a pair of horizontal lines (viewed binocularly or monocularly) produced much smaller VPEL shifts. Because the perceived pitch of the pitched horizontal line display was nearly the same as the perceived pitch of the pitched vertical line and dot array, the relatively small influence of pitched horizontal lines on VPEL cannot be attributed simply to an underestimation of their pitch. In Experiment 2, the effects of pitched vertical lines, dots, and horizontal lines on VPEL were again measured, together with their effects on resting gaze direction (in the vertical dimension). As in Experiment 1, vertical lines and dots caused much larger VPEL shifts than did horizontal lines. The effects of the displays on resting gaze direction were highly similar to their effects on VPEL. These results are consistent with the hypothesis that VPEL shifts caused by pitched visual arrays are due to the direct influence of these arrays on the oculomotor system and are not mediated by perceived pitch.
\end{abstract}

It is well known that pitched visual displays alter the apparent visual horizon - that is, the locus that appears to correspond to eye level (e.g., Matin \& Fox, 1989). This locus is often referred to as visually perceived eye level (VPEL). In general, top-toward and top-away pitches of a stimulus relative to the observer cause upward and downward VPEL shifts, respectively, of approximately $40 \%-50 \%$ of the pitch angle. This pattern of results has been found for a wide variety of pitched stimuli, ranging from complex fullfield scenes (e. g., Matin \& Fox, 1989; Stoper \& Cohen, $1989)$ to a single pitched vertical luminous line viewed in an otherwise dark setting (Matin \& Li, 1992a, 1994a).

Given the reliability and robustness of this effect, it is interesting that a pitched pair of luminous horizontal lines has little or no influence on VPEL (Matin \& Li, 1994a). This finding has been interpreted by Matin and his colleagues in terms of the great circle model, a geometrical analysis demonstrating that the retinal images of pitched vertical line stimuli viewed by an eye looking straight

The authors are grateful to Malcolm Cohen and Arnold Stoper for discussions of the research and to Kristin Caufield for assistance with data collection. The authors also thank Len Matin for his thoughtful and thorough review of the submitted manuscript. Correspondence should be addressed to R. B. Post, Department of Psychology, University of California, Davis, CA 95616 (e-mail: rbpost@ucdavis.edu). ahead resemble those that would be formed by vertical stimuli if the eye were to be rotated upward or downward (e. g., Matin \& Li, 1992a). That is, in each case, the line stimuli fall on great circles that intersect on the central vertical meridian (CVM) of the eye. Thus, according to this model, pitched horizontal lines fail to produce VPEL shifts because they do not lie on great circles that intersect on the CVM (Matin \& Li, 1992b).

Not only do pitched horizontal lines fail to coincide with great circles that converge on the CVM, but they provide little or no other information about their pitched orientation relative to the observer. That is, while vertical lines contain prominent linear perspective cues with respect to their pitch, horizontal lines do not. Additionally, if viewing is monocular, as it was in the study by Matin and Li (1994a), other important pitch information (i.e., convergence, binocular disparity) is also absent. In short, the pitched horizontal lines viewed by the subjects in Matin and Li's (1994a) experiment may have been perceived as much less pitched than the pitched vertical lines. Perhaps it is for this reason, rather than the great circle model, that pitched horizontal lines have such a small effect on VPEL. The aim of the present experiments was to test this possibility.

Experiment 1 examined the effects of pitched visual arrays on both VPEL and perceived pitch. In four separate 
conditions, subjects made VPEL judgments while binocularly viewing (1) a pair of pitched vertical lines, (2) a pair of pitched horizontal lines, and (3) a pitched circular array of dots. In a fourth condition, they viewed the pitched vertical line stimulus monocularly. The three visual arrays are depicted in Figure 1. These stimuli were selected, in part, because they varied the nature of the information or cues available to subjects about the pitch of the stimuli (see Cutting \& Vishton, 1995, for a recent review of this topic). As seen in Table 1, binocular disparity and convergence were available in all stimulus conditions except for the monocularly viewed horizontal lines, whereas accommodative cues were present for all of the conditions. Linear perspective, as defined by the presence of converging lines in the projection, was present for the vertical line stimulus. Such lines are not present in the horizontal line stimuli, but imaginary lines connecting the endpoints of the horizontal lines would show similar convergence. This form of implied linear perspective is represented by question marks in Table 1. Finally, relative density (texture gradient) information was present only for the dot array. The latter stimulus is of particular interest in the context of the great circle model because it (as with the horizontal line array) contains no lines that fall on great circles intersecting on the CVM and, thus, according to the great circle model, should fail to produce a VPEL shift, despite the presence of rich information about pitch.

\section{EXPERIMENT 1}

\section{Method}

Nine male and 22 female volunteers (19-42 years of age) served as subjects. All had 20/20 visual acuity either naturally or by means of contact lenses. (It was important to avoid spectacle frames since these would have occluded portions of the displays). With one exception (the senior author, R.B.P.), all of the subjects were experimentally naive. The displays used in this experiment (see Figure 1) were constructed of luminous tape mounted on a board that could be pitched about its center. One display (Figure 1A) consisted of a pair of vertical lines, $46.6 \mathrm{~cm}$ long and $0.2 \mathrm{~cm}$ wide, separated by a distance of $31.3 \mathrm{~cm}$. At the $33-\mathrm{cm}$ viewing distance, the lines subtended $65.0^{\circ}$ vertical $\times 0.3^{\circ}$ horizontal, with a line separation of $50.7^{\circ}$ at the center of the display when presented in the vertical orientation. When pitched, the angular sizes of this array changed somewhat as a result of the changed viewing distance. A second display (Figure 1B) was identical to the first, with the exception that its orientation was rotated $90^{\circ}$, to create a horizontal line stimulus. A third display (Figure 1C) consisted of 66 dots randomly located within a circular field $43.5 \mathrm{~cm}$ in diameter. Each dot had a 0.6- $\mathrm{cm}$ diameter, making the total area of the dots the same as the total area of the line stimuli. The field on which the dots were mounted was equal in area to that enclosed by the outer dimensions of the line stimuli. All displays were centered on the subjects' median plane for both binocular and monocular viewing conditions, and the axis of display rotation was at objective eye level. Display luminance was approximately $1.4 \mathrm{cdm}^{-2}$ when the room lights were extinguished, but it decreased with time in the dark as the luminous tape gradually lost its brightness. Since this loss in stimulus intensity was paralleled by increasing dark adaptation, the stimuli remained easily visible throughout the experiment. A plastic rod, $14.6 \mathrm{~cm}$ long and $0.6 \mathrm{~cm}$ in diameter, was mounted on the right side of the table on which the displays were positioned. The rod was rotatable in the pitch dimension, and its bottom end was attached to a potentiometer that provided a voltage proportional to the pitch angle.

VPEL measures. VPEL measures were collected from 21 subjects, each of whom was seated with his/her head stabilized by a bite bar so that Reid's baseline ${ }^{1}$ was perpendicular to the gravity vector and the eyes were at the same height as the center of the display. A spot of light, $0.5 \mathrm{~cm}$ in diameter $\left(0.3^{\circ}\right.$ of visual arc), was projected from a low-power laser onto the back side of a translucent panel midway between the left and right edges of the display. The translucent panel displayed to the subject the back-illuminated spot, which was located at the same distance as the display. The subjects directed the experimenter to adjust the vertical position of the spot until it appeared to be at their VPEL. This position was recorded by the experimenter from a calibration scale on the back of the panel. Five judgments were made for each of three pitches of the stimulus display $\left(20^{\circ}\right.$ top-toward, upright, and $20^{\circ}$ top-away). Responses were made with the spot starting alternately above and below the subjects' (presumed) VPEL. Order of the three pitches was random-
(A)

(B)
(C) Dots

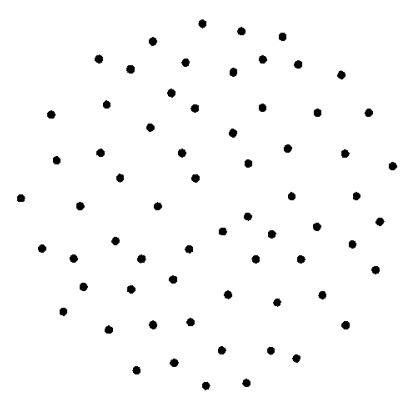

Horizontal 
Table 1

Perceived Pitch Cues Available for Each Display in Experiment 1

\begin{tabular}{lcccc}
\hline & \multicolumn{4}{c}{ Stimulus Display } \\
\cline { 2 - 5 } \multicolumn{1}{c}{ Cue } & Vertical Lines & Dots & Binocular & Monocular \\
\cline { 2 - 5 } Binocular disparity & $\times$ & $\times$ & $\times$ & \\
Ocular convergence & $\times$ & $\times$ & $\times$ & \\
Accommodation & $\times$ & $\times$ & $\times$ & $\times$ \\
Linear perspective & $\times$ & & $?$ & $?$ \\
Relative density & & & & \\
(Texture gradient) & \multicolumn{4}{c}{$\times$} \\
\hline
\end{tabular}

Note $-x=$ cue is present. ? = cue may be present.

ized across subjects. Viewing was binocular for the vertical line and random dot stimuli and was either binocular or monocular for the horizontal line stimulus.

Perceived pitch measures. Measures of perceived pitch were collected from 10 subjects under viewing conditions that were very similar to those of the VPEL measures. The laser spot was projected onto the translucent panel in the center of the display, midway between the left and right edges of the display at objective eye level. The subjects were instructed to fixate the spot and to adjust the pitch of an unseen rod with the unseen right hand to match the perceived pitch of the display. The subjects indicated when they had completed this task for a given stimulus, and the experimenter then sampled by means of a computer the voltage associated with the rod pitch. This voltage was converted to the pitch of the rod and displayed for the experimenter to record. The subjects then made a verbal estimate of the perceived pitch of the display, in terms of both the number of degrees that the display appeared pitched from vertical and whether the top appeared pitched toward or away from them. These responses were to serve as a "screening device" to replace any subjects whose verbal and manual pitch estimates were not highly correlated. (This, however, never occurred.) Measures were collected for each of the displays, which were placed at 11 different pitches (corresponding to every $5^{\circ}$ between $25^{\circ}$ top-toward and $25^{\circ}$ top-away). Stimuli were positioned while the subjects' eyes were shut to preclude the possibility of viewing the displays in room illumination. This procedure helped ensure that responses were based on the perceptual characteristics of the stimuli rather than some other basis. All pitches for one display were completed before proceeding to the next. The order of displays and pitches was randomized.

\section{Results}

VPEL. The median was calculated for the five responses made by each subject for each stimulus/pitch combination. The means of these values are shown in the top panel of Figure 2 for each stimulus display. It can be seen from the figure that, for both the vertical line and the dot displays, positive (top-toward) and negative (top-away) pitches elevated and lowered VPEL, respectively, apparently by the same amount. However, for the horizontal display, viewed monocularly or binocularly, the effects of pitch were severely (and equally) attenuated.

Perceived pitch. Each subject's manual and verbal estimates of perceived pitch were compared for each display by means of linear regression. For all subjects, the two measures were highly correlated (all $r s>90$ ). Additionally, both manual and verbal estimates were regressed against stimulus pitch separately for each of the stimuli. The slopes and intercepts obtained from these regressions are presented in Table 2. It is apparent from this table that the results from the manual and verbal measures were highly similar. Because of this strong agreement, together with somewhat less variability in the manual measures, only the latter were used in the subsequent analyses.

The means for all subjects' manual responses for each display are shown in the bottom panel of Figure 2. It is clear from the figure that perceived pitch varied systematically with stimulus pitch. Additionally, there seem to be slight differences among the four stimuli in the degree to which they were perceived as being pitched. Specifically, the vertical line and dot stimuli appear to have generated somewhat greater perceived pitch changes (particularly for the top-away pitches) than did the horizontal lines, whether the latter were viewed monocularly or binocularly.

To compare the effects of the four stimulus conditions on VPEL and perceived pitch, regressions of VPEL and of perceived pitch on stimulus pitch were calculated separately for each subject for each of the four stimuli. The slopes and intercepts resulting from these regressions are presented in the bottom portiun of Table 2. The slope coefficients for the fitted regressions were then entered into a 2 (measure: VPEL vs. perceived pitch) $\times 4$ (stimulus) analysis of variance (ANOVA). The effect of measure was statistically significant $[F(1,29)=95.1, p<.001]$, representative of the fact that the mean slope of perceived pitch regressed on stimulus pitch was greater than that of VPEL (see Figure 2). In other words, changes in stimulus pitch produced a greater change in perceived pitch than they did in VPEL. The effect of stimulus condition was also statistically significant $[F(3,87)=23.7, p<$ $.001]$. Concerning the latter effect, planned comparisons failed to reveal either a significant difference between the results for the vertical line and dot stimuli or a difference between monocular and binocular viewing of the horizontal line stimulus. However, the results for horizontal lines, viewed monocularly or binocularly, were significantly different from those obtained with either the vertical lines or dot array (all $p \mathrm{~s}<.001$ ). The measure $\times$ stimulus interaction was not statistically significant $[F(3,87)<1.00]$.

It is apparent from Figure 2 that the difference between the vertical line and dot stimuli on the one hand and the horizontal line stimuli on the other is proportionally greater for the VPEL measures (top panel) than for the perceived pitch measures (bottom panel). That is, although horizontal lines generate somewhat less perceived pitch than do the vertical line and dot stimuli, their influence on VPEL is only a small fraction of the effect of vertical line and dot stimuli on VPEL. This difference among stimuli in their effects on perceived pitch and VPEL can be expressed by taking the ratios: (slope of [perceived pitch/ stimulus pitch])/(slope of [VPEL/stimulus pitch]). The resulting values for the case of vertical lines and binocularly viewed horizontal lines are 2.6 and 17.0 , respectively. To highlight the proportional differences between the two response measures, the slope coefficients for both perceived pitch and VPEL data are plotted adjacently 

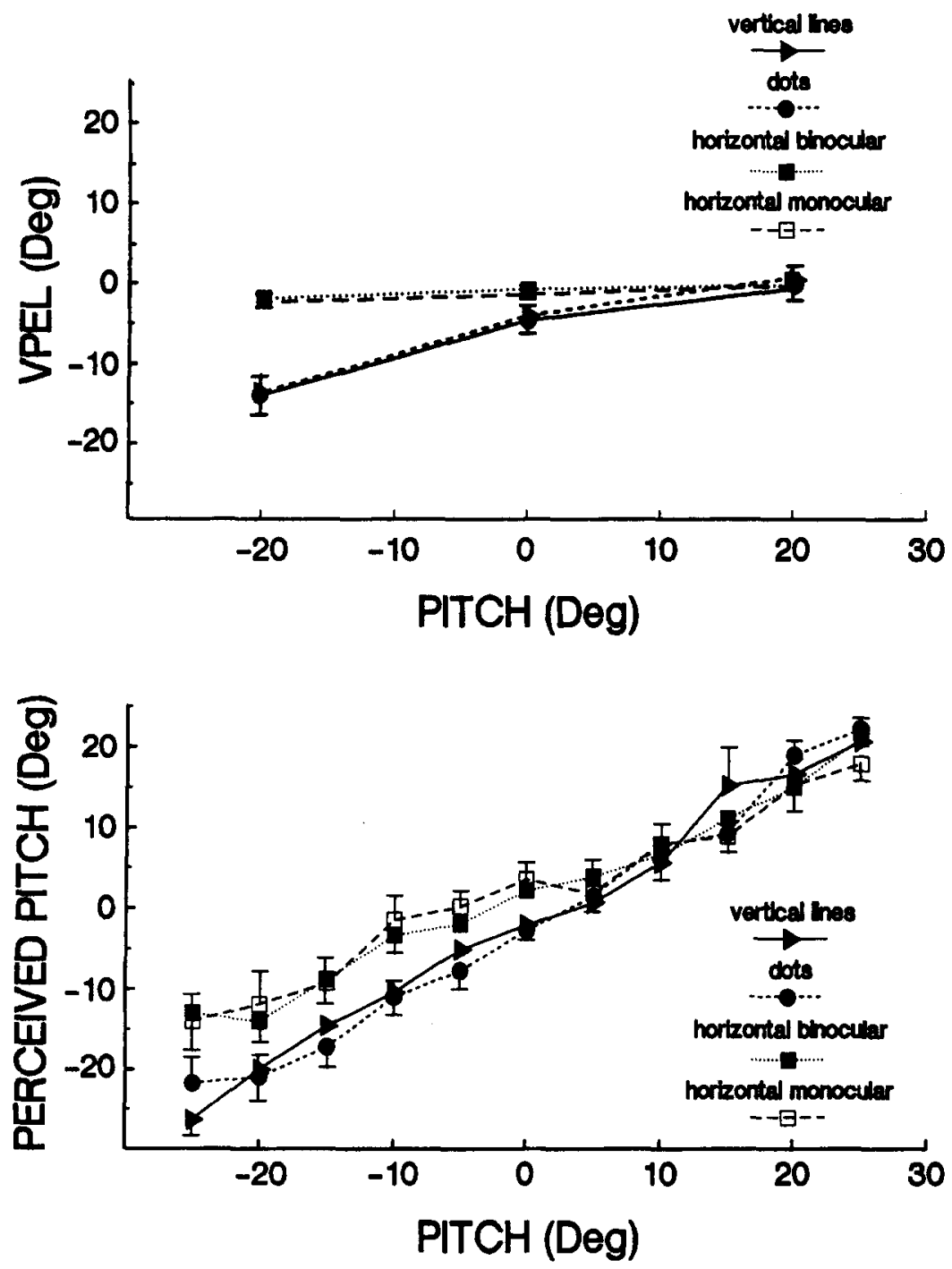

Figure 2. Top panel: Mean VPEL levels measured while viewing the vertical line stimulus (triangles), the random dot stimulus (circles), or the horizontal line stimulus, either binocularly (solid squares) or monocularly (open squares). Bottom panel: Mean perceived pitch measured while viewing each of the stimuli. Negative numbers on the abscissa refer to top-away pitches, and positive numbers refer to top-toward pitches. Error bars correspond to $\pm 1 S E M$. To avoid clutter, not all error bars are included. Some error bars fall within the markers.

in Figure 3, with the scales adjusted so that the bars corresponding to the vertical line stimulus are identical. In this figure it is again apparent that the values for both VPEL and perceived pitch measures of the horizontal line stimuli are less than those for the vertical line and dot stimuli. More important, however, is the fact that the relative ineffectiveness of the horizontal line stimulus in causing VPEL changes is far greater than its ineffectiveness in eliciting perceived pitch changes.

\section{Discussion}

Experiment 1 demonstrated that pitched vertical lines produce substantial shifts in VPEL, replicating the results of previous studies (e.g., Matin \& Fox, 1989; Matin \& Li, 1992b, 1994a; Post \& Welch, 1996; Stoper \& Cohen, 1989). Furthermore, a pitched array of dot stimuli generated shifts in VPEL equal to those for pitched vertical lines. However, pitched horizontal lines, whether viewed monocularly or binocularly, had only a small percentage of the effect on VPEL that occurred for pitched vertical lines or dots. The finding for monocularly viewed horizontal lines essentially replicates Matin and Li (1994a). The results for the perceived pitch measures were similar to those for the VPEL measures in that both monocularly and binocularly viewed horizontal lines are relatively less effective in causing changes in perceived pitch 
Table 2

Slopes and Intercepts for Regressions of Manual and Verbal Measures of Perceived Pitch and VPEL Measures Against Stimulus Pitch for Each Display in Experiment 1

\begin{tabular}{|c|c|c|c|c|}
\hline \multirow[b]{3}{*}{ Measure } & \multicolumn{4}{|c|}{ Stimulus Display } \\
\hline & \multirow{2}{*}{$\begin{array}{c}\text { Vertical } \\
\text { Lines }\end{array}$} & \multirow[b]{2}{*}{ Dots } & \multicolumn{2}{|c|}{ Horizontal Lines } \\
\hline & & & Binocular & Monocular \\
\hline \multicolumn{5}{|c|}{ Perceived Pitch (Manual) } \\
\hline Slope & .93 & .92 & .68 & .62 \\
\hline Intercept & -1.84 & -2.01 & 1.67 & 1.69 \\
\hline \multicolumn{5}{|c|}{ Perceived Pitch (Verbal) } \\
\hline Slope & .92 & .92 & .63 & .69 \\
\hline Intercept & -0.61 & -0.72 & 3.25 & 2.88 \\
\hline \multicolumn{5}{|l|}{ VPEL } \\
\hline Slope & .36 & .33 & .04 & .05 \\
\hline Intercept & -5.72 & -6.53 & -1.09 & -1.46 \\
\hline
\end{tabular}

than are vertical lines and dot arrays. However, proportionally speaking, the ineffectiveness of horizontal lines with respect to perceived pitch change was substantially less than their ineffectiveness in causing VPEL shifts.

It is perhaps surprising that monocularly viewed horizontal lines generated as strong a perception of pitch as they did (about $67 \%$ of the pitch experienced with the binocularly viewed vertical lines). Previous work has suggested that linear perspective is a far more effective cue to stimulus pitch than compression gradients (e.g., Braunstein \& Payne, 1969; Gillam, 1970; Rosinski \& Levine, 1976). Whereas the vertical line stimulus in the present experiment generated linear perspective, the horizontal line stimulus contained only implied linear perspective at the endpoints of the lines and failed completely to gen- erate a compression gradient because it contained only two lines. However, viewing conditions in each of the prior studies included an aperture that blocked from view the edges of the stimuli. Therefore, the implied linear perspective at the endpoints of the present stimulus was not available in prior studies. Additionally, the subjects in the present experiment had accommodation available as a cue with the horizontal line stimulus. The prior work of Braunstein and Payne (1969) and Rosinski and Levine (1976) used two-dimensional projections of pitched stimuli, and, therefore, accommodation would have actually conflicted with the perception of pitch. Additionally, the displays used by Gillam (1970) were viewed at a distance of $98 \mathrm{~cm}$, where accommodation may not have been as effective a cue to slant as it was in the present experiment.

The results of Experiment 1 produced no evidence that the reason why pitched horizontal lines produce only weak VPEL shifts is simply because they do not look very pitched. This conclusion is consistent with the findings of several other studies that were reported while the present research was underway. Hudson, Li, and Matin (1996) noted that individual differences in the perceived pitch of displays and the degree to which they alter VPEL are uncorrelated. Additionally, Li and Matin (1998) found that, although an eccentric parallel two-line stimulus tilted clockwise or counterclockwise in the frontoparallel plane causes a shift in VPEL, it is nevertheless correctly perceived as upright, rather than pitched. Servos, Matin, and Goodale (1995) found that selective brain damage can apparently force a dissociation between VPEL and perceived pitch, as evidenced by a brain-damaged patient suffering from visual form agnosia for whom the

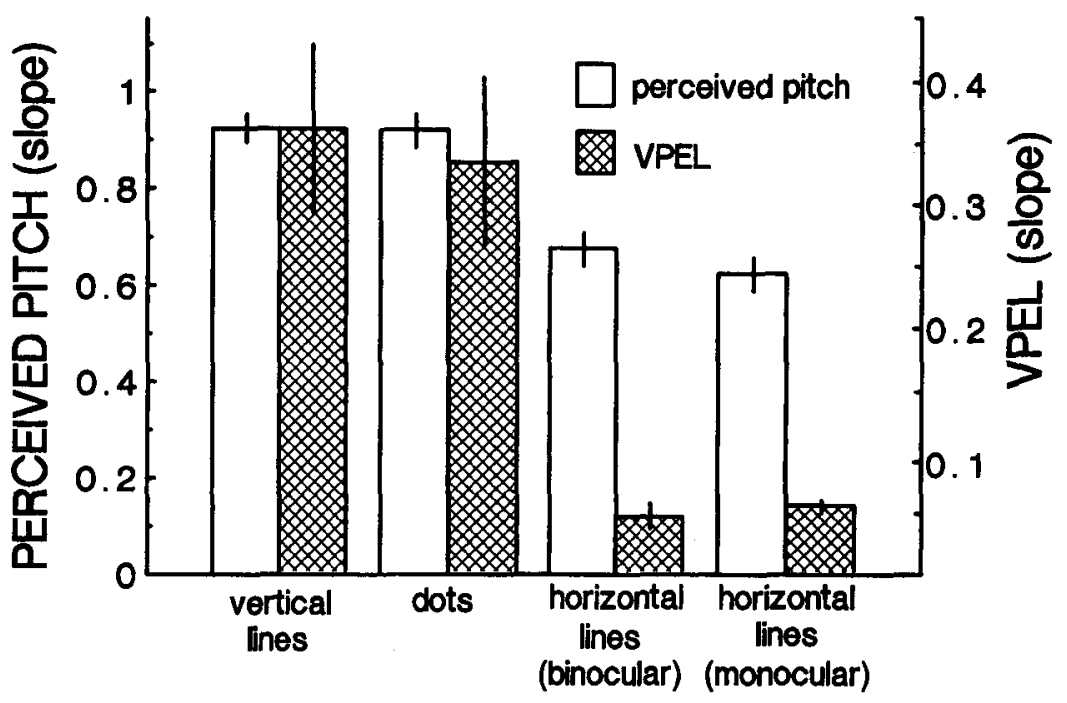

STIMULUS

Figure 3. Relative effects for both VPEL and perceived pitch measures for each stimulus. Bars correspond to the slope coefficients obtained for linear regressions fitted to each of the curves in Figure 2. Error bars correspond to $\pm 1 S E M$. 

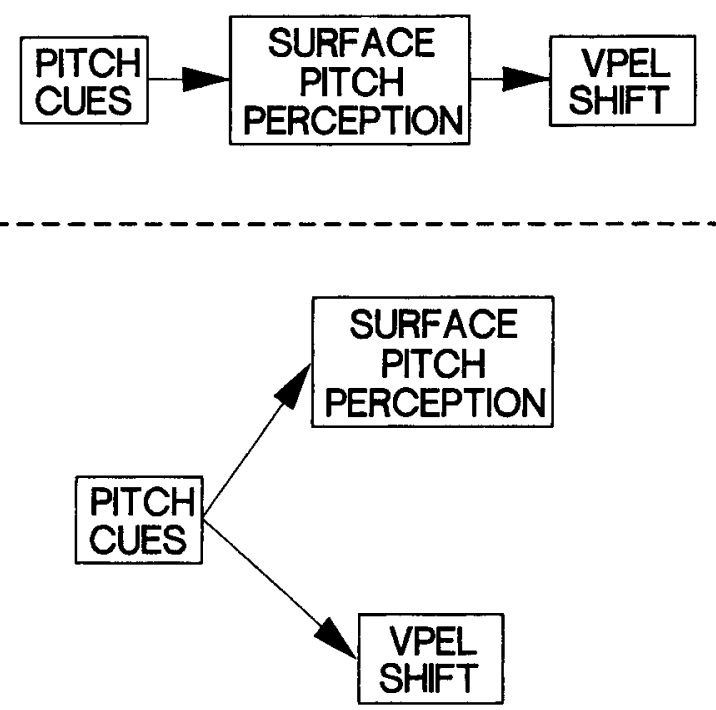

Figure 4. Top panel: Serial model in which pitch cues determine surface pitch perception, which in turn determines VPEL. Bottom panel: Parallel model in which pitch cues contribute to surface pitch perception and VPEL independently.

perceived pitch of pitched displays was severely impaired while, at the same time, VPEL was biased in the expected manner.

Taken together, these studies (and ours) suggest that VPEL shifts are not the direct result of perceived surface pitch. Rather, stimulus pitch information appears to contribute independently to VPEL shifts and pitch percepiion. These alternate conceptions of the relationship between perceived pitch and VPEL shifts are depicted schematically in Figure 4 . The finding by Hudson et al. (1996) that individual differences in pitch perception and VPEL shifts are uncorrelated suggests that, once pitch cues are initially processed, additional factors operate on either pitch perception or VPEL (or both) but that these factors are independent of one another. $\mathrm{Li}$ and Matin's (1998) finding that a two-line stimulus tilted clockwise or counterclockwise in the frontoparallel plane can influence VPEL while simultaneously being (correctly) seen as upright is consistent with the possibility that such lines can be initially processed as a pitch cue that allows them to give rise to VPEL shifts. They do not actually appear pitched however, because pitch perception requires the presence of other information as well. Finally, the form agnosia observations of Servos et al. (1995) strongly suggest that the areas of the brain involved in the processing of pitch cues for the purpose of determining perceived pitch can be damaged separately from the areas involved in the subsequent processing of pitch cues for their influence on VPEL.

As noted previously, the effects of pitched vertical lines on VPEL have been explained by Matin and his colleagues (e.g., Matin \& Li, 1992a) in terms of the great circle model. Recall that, according to this model, pitched vertical lines form retinal images consistent with those that would occur if the lines were upright and the eye was pitched. In both cases, the line stimuli fall on great circles that intersect on the CVM of the eye. In contrast, pitched horizontal lines do not lie on great circles that intersect the CVM (Matin \& Li, 1992b) and, therefore, do not generate strong VPEL shifts. This being the case, it is very interesting that the dot display used in the present experiment elicited VPEL shifts similar to those caused by pitched vertical lines. That is, the great circle model would seem to predict that the dot display would be ineffective in causing VPEL shifts because it does not include vertical contours. However, a possible reconciliation of the present findings with this model may lie in the fact that, at the left and right edge of each dot, the contour orientation is vertical and remains approximately vertical for a short distance in either direction (see Figure 1C). Since Li and Matin (1991) and Matin and Li (1994b) have shown that the effects of pitch are additive along the length of a single vertical line (or along two widely separated vertical lines) as extent is increased, perhaps the present results obtained with the dot array are another example of such additivity.

\section{EXPERIMENT 2}

Pitched visual arrays not only bias VPEL in the direction of the pitch but also cause a corresponding shift in the direction of gaze when subjects attempt to look at the (unseen) horizon or even when they are instructed simply to relax the eyes and allow them to drift to their natural resting position (M. M. Cohen, Ebenholtz, \& Linder, 1995; Matin \& Li, 1995). M. M. Cohen et al. (1995) refer to the latter effect as the optostatic response. Thus, visual pitch is accompanied by an involuntary rotation of the eyes such that the line of regard is more nearly perpendicular to the viewed surface. Therefore, perhaps the ineffectiveness of pitched horizontal lines in producing a VPEL shift is due to (or at least accompanied by) their relative ineffectiveness in altering the direction of relaxed gaze. Experiment 2 examined this possibility.

\section{Method}

Three male and 7 female volunteers ( $21-42$ years of age), with $20 / 20$ visual acuity (naturally or corrected by means of contact lenses), served as subjects in all conditions of the experiment. Nine subjects were naive with respect to the experimental questions and hypotheses; 1 subject (the senior author, R.B.P.) was not.

VPEL measures. The stimulus displays and procedure for measuring VPEL were the same as in Experiment 1. Because there were no differences in the effects of the monocular and binocular viewing conditions of the horizontal displays in Experiment 1, only the vertical line, dot, and binocularly viewed horizontal display conditions were examined. Measures were obtained for five display pitches (every $10^{\circ}$ from $20^{\circ}$ top-toward to $20^{\circ}$ top-away).

Gaze direction measures. The subjects' vertical gaze direction was measured in this experiment by means of a subjective positive afterimage (AI) technique similar to that of Leibowitz, Shupert, Post, and Dichgans (1983). A foveal AI was generated by having the subjects fixate the center of a photoflash that was masked to expose a square area $(0.5 \mathrm{~cm}$ on a side). At a stimulation distance of $50 \mathrm{~cm}$, 
the resulting AI subtended $0.57^{\circ} \times 0.57^{\circ}$. After forming the AI, the room lights were extinguished, and the subjects viewed the same displays used for the VPEL measures. They were instructed to look at the center of a given display in the horizontal dimension and to relax and make no conscious effort to look in any particular direction in the vertical dimension. After approximately $30 \mathrm{sec}$, the display was briefly illuminated with a flashlight (typically less than $5 \mathrm{sec}$ ), and the subjects saw both their AI and a calibration scale. The calibration scale consisted of a column of numbers that extended the height of the display. It was attached to the front surface of the display and was centered in the horizontal dimension. Because of the location of the calibration scale, the AI appeared superimposed on it. The subjects then read out loud the number of the scale against which the AI appeared to be located, and the experimenter recorded this value. On some trials, the AI was visible before the flashlight was turned on; on other trials, it had faded. If the AI had faded, it always reappeared when the flashlight was turned on. Five such measures were recorded for each stimulus/pitch combination.

\section{Results}

VPEL. The median was calculated for the five responses made by each subject for each stimulus/pitch combination. The means of these values are shown in the top panel of Figure 5 for each stimulus display. As in Experiment 1 , pitching the vertical line and dot displays re-
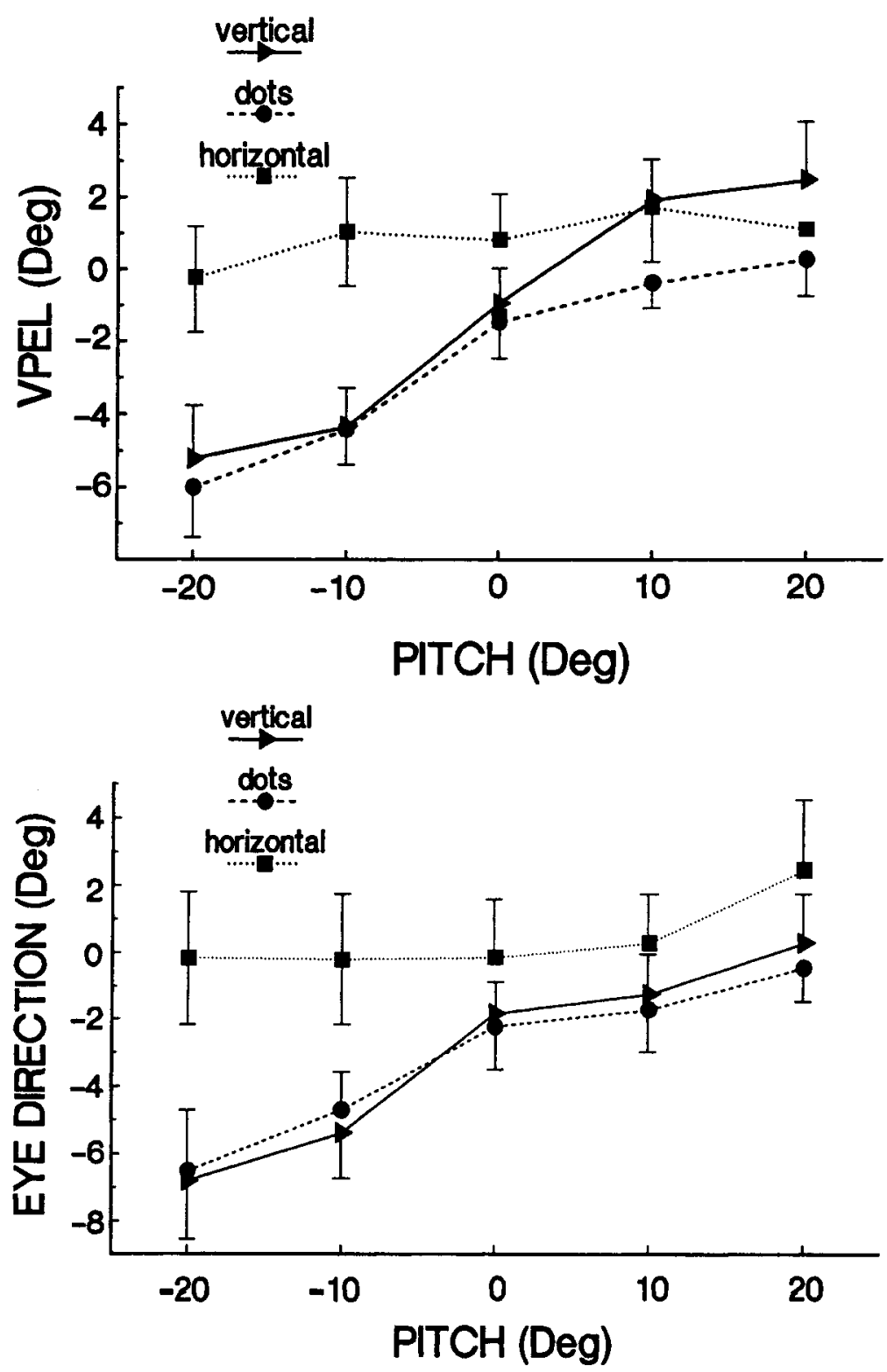

Figure 5. Top panel: Mean VPEL levels measured while viewing the vertical line stimulus (triangles), the random dot stimulus (circles), or the horizontal line binocularly (squares). Bottom panel: Mean resting eye direction measured while viewing each of the stimuli. Negative numbers on the abscissa refer to top-away pitches, and positive numbers refer to top-toward pitches. Error bars correspond to $\pm 1 S E M$. To avoid clutter, not all error bars are included. 
sulted in substantial changes in VPEL, whereas the effects of the horizontal display were minimal.

Gaze direction. The median of the five responses made by each subject for each stimulus/pitch combination was determined, and the means of these values are shown in the bottom panel of Figure 5. These results appear highly similar to the VPEL results shown in the top panel of the figure.

To compare the effects of the three stimulus conditions on VPEL and gaze direction, regressions of VPEL and gaze direction on stimulus pitch were calculated separately for each subject for each of the three stimuli. The slope coefficients for the fitted regressions were then entered into a 2 (measure: VPEL vs. gaze direction) $\times 3$ (stimulus) ANOVA. Neither the effect of measure nor the measure $X$ stimulus interaction was statistically significant $[F(1,9)<$ 1.00 , and $F(2,18)<1.00$, respectively]. However, the effect of stimulus was statistically significant $[F(2,18)=$ $15.0, p<.001]$. Planned comparisons indicated that the results for the vertical line stimulus were statistically significantly different from those obtained with the horizontal line stimulus $[F(1,9)=68.41, p<.001]$, but not statistically different from those obtained with the dot stimulus $[F(1,9)=3.55, p>.05]$. The results for the dots were also significantly different from those obtained with the horizontal stimuli $[F(1,9)=8.06, p<.05]$.

\section{Discussion}

As was the case in Experiment 1 and the study of Matin and Li (1994a), the shifts in VPEL caused by pitched horizontal lines were only a small fraction of those obtained with pitched vertical lines. Also similar to Experiment 1, the dot stimulus had a greater influence on VPEL than did the horizontal line stimulus.

The effects of the vertical line and dot stimuli on vertical gaze direction are similar to those reported previously for viewing a large patterned stimulus (M. M. Cohen et al., 1995; Matin \& Li, 1995). The highly similar VPEL and vertical gaze direction results suggest that it is because pitched horizontal lines have only a minimal influence on vertical gaze direction that they have so little effect on VPEL. It should be remembered, however, that this correlation between VPEL and gaze direction data does not necessarily imply a causal relationship between the two events.

\section{GENERAL DISCUSSION}

The obvious question raised by the present results is this: Why is it that, while pitched vertical lines (Figure 1A) and equally pitched horizontal lines (Figure 1B) are perceived as pitched by roughly the same amount, they differ so substantially in their effects on VPEL? One possible explanation is that, although both of these stimulus configurations contain the perspective cues necessary for the experience of visual pitch, the vertical contours elicit a much greater optostatic response (as demonstrated in our Experiment 2). This interpretation of the present results is congruent with the claim by M. M. Cohen et al. (1995) that VPEL shifts are caused by (rather than a correlate of) the optostatic response. One reason why horizontal contours might elicit little or no optostatic response is the fact that any such tendency of the eyes to move orthogonally with respect to the contours produces retinal slip, which is immediately nullified by optokinetic nystagmus $(\mathrm{OKN})$. When retinal slip is produced by moving stimuli, it typically elicits $O K N$ as a means of nullifying or minimizing it (see, e.g., B. Cohen, Matsuo, \& Raphan, 1977; Collewijn, 1985). It may be proposed that in the present situation of viewing stationary pitched horizontal lines, the initial optostatic response would cause retinal slip similar to that produced by horizontal lines moving in the vertical direction. This slip would in turn elicit $\mathrm{OKN}$ in the direction opposite the optostatic response, and, therefore, the two eye-movement tendencies would cancel each other. The net effect of this interaction would therefore be little or no observed optostatic response. Clearly, vertical contours pose no such obstacle to the optostatic response since they are parallel to the direction of the response and would thus not generate substantial retinal slip. Therefore, the optostatic response could occur unhindered and lead, in turn, to the substantial effect on VPEL that we (and others) have observed. This analysis predicts that viewing a pitched array that is composed of both vertical and horizontal contours (e.g., a checkerboard pattern) will result in a reduced optostatic response (relative to that caused by a stimulus containing only vertical lines) and that observers will undergo a commensurate difference in the VPEL shift elicited by these two arrays. We are currently investigating this possibility.

\section{REFERENCES}

Braunstein, M. L., \& Payne, J. W. (1969). Perspective and form ratio as determinants of relative slant judgments. Journal of Experimental Psychology, 81, 584-590.

Cohen, B., Matsuo, V., \& Raphan, T. (1977). Quantitative analysis of the velocity characteristics of optokinetic nystagmus and optokinetic after nystagmus. Journal of Physiology, 270, 321-344.

Cohen, M. M., Ebenholtz, S. M., \& Linder, B. J. (1995). Effects of optical pitch on oculomotor control and the perception of target elevation. Perception \& Psychophysics, 57, 433-440.

Collewiun, H. (1985). Integration of adaptive changes of optokinetic reflex, pursuit and the vestibulo-ocular reflex. In A. Berthoz \& G. Melvill Jones (Eds.), Adaptive mechanisms in gaze control (pp. 51-69). New York: Elsevier.

Cutting, J. E., \& Vishton, P. M. (1995). Perceiving layout and knowing distances: The integration, relative potency, and contextual use of different information about depth. In W. Epstein \& S. Rogers (Eds.), Perception of space and motion (pp. 69-117). San Diego: Academic Press.

GillaM, B. (1970). Judgments of slant on the basis of foreshortening. Scandinavian Journal of Psychology, 11, 31-34.

Hudson, T., LI, W., \& Matin, L. (1996, November). Independence of visually perceived eye level and perceived pitch. Paper presented at the annual meeting of the Psychonomic Society, Chicago.

Leibowitz, H. W., Shupert, C. L., Post, R. B., \& Dichgans, J. (1983). Autokinetic drifts and gaze deviation. Perception \& Psychophysics, 33, 455-459.

LI, W., \& MATIN, L. (1991). Spatial summation of influences on visually 
perceived eye level from a single variably-pitched 1-line stimulus. Investigative Ophthalmology \& Visual Science, 32(Suppl.), 1272.

LI, W., \& Matin, L. (1998). Change in visually perceived eye level without change in perceived pitch. Perception, 27, 553-572.

Matin, L., \& Fox, C. R. (1989). Visually perceived eye level and perceived elevation of objects: Linearly additive influences from visual field pitch and from gravity. Vision Research, 29, 315-324.

Matin, L., \& LI, W. (1992a). Mislocalizations of visual elevation and visual vertical induced by visual pitch: The great circle model. In B. Cohen, D. L. Tomko, \& F. E. Guedry (Eds.), Sensing and controlling motion: Vestibular and sensorimotor function (Annals of the New York Academy of Sciences, Vol. 656, pp. 242-265). New York: New York Academy of Sciences.

Matin, L., \& LI, W. (1992b). Visually perceived eye level: Changes induced by a pitched-from-vertical 2-line visual field. Journal of Experimental Psychology: Human Perception \& Performance, 18, 257-289.

Matin, L., \& LI, W. (1994a). The influence of a stationary single line in darkness on the visual perception of eye level. Vision Research, 34, 311-330.

Matin, L., \& LI, W. (1994b). Spatial summation among coextensive and parallel line segments across wide separations $\left(50^{\circ}\right)$ : Egocentric localization and the great circle model. Vision Research, 34, 2577-2598.
Matin, L., \& LI, W. (1995). Multimodal basis for egocentric spatial localization and orientation. Journal of Vestibular Research, 5, 499-518.

Post, R. B., \& WELCH, R. B. (1996). The role of retinal versus perceived size in the effects of pitched displays on visually perceived eye level. Perception, 25, 853-859.

Rosinski, R. R., \& Levine, N. P. (1976). Texture gradient effectiveness in the perception of surface slant. Journal of Experimental Child Psychology, 22, 261-271.

Servos, P., Matin, L., \& Goodale, M. A. (1995). Dissociation between two modes of spatial processing by a visual form agnostic. NeuroReport, 6, 1893-1896.

STOPER, A. E., \& COHEN, M. M. (1989). Effect of structured visual environments on apparent eye level. Perception \& Psychophysics, 46, 469-475.

\section{NOTE}

1. Reid's baseline is the imaginary line connecting the outer canthus of the eye to the auditory canal.

(Manuscript received June 3, 1998; revision accepted for publication March 18, 1999.) 